gational problem. The timing precision is easier to obtain, particularly with electrically fired airguns or explosions.

In recent years advances in marine seismic work have come more from new methods of quantitative analysis and numerical modelling than of experimental technique. An example of such an analytical method is that used by Stoffa and Buhl to extract phase velocity information from the record sections. This is done on a shot by shot basis using a semblance technique to determine the phase velocity having the greatest coherence across each set of 24 traces beginning in turn at each successive digitised sample along the nearest or first trace. The technique is useful in that it enables over-lapping or 'crossing-over' signals of different phase velocity to be separated as well as giving an objective assessment of the onset times of second arrivals which might otherwise have been picked too late. From analysis of the semblance plots a precise travel time versus distance plot is produced for all arrivals whose semblance coefficient exceeds a given value, and this plot in turn is inverted using a derivation of a standard method to give a highly detailed velocity/depth profile of the oceanic crust.

A disadvantage of the ESP method outlined above is that the structure under each shot and receiver position may differ, whereas for a fixed ocean-bottom seismograph at least half this possible lateral variation is avoided. However some indication of lateral velocity inhomogeneity can be obtained, again using two ships, by keeping the source and receiver at a fixed distance (constant of fset profile or COP) appropriate to the interface of interest. By combining COPs having different offset distances with CDP and normal multichannel reflection profiles it is possible to approach the ideal deep crustal seismic experiment. However to model the Earth fully taking account of all the constraints provided by these types of observations is probably taking the problem beyond the scope of the best computer facilities.

The technique described by Stoffa and Buhl opens up exciting possibilities for detailed deep crustal studies in the oceans. In practice however it is unlikely that there will be many researchers in a position to follow their example. Regrettably, multichannel digital recording systems, digital seismic reflection processing and two-ship experiments are expensive. But such experiments might continue perhaps on an inter-institution basis in areas of mutual scientific interest. Continental margins in particular, where the results could be of more than academic interest and where two-ship experiments are more likely to obtain the necessary funding would provide attractive targets. Such an approach has already led to a joint FrenchUnited Kingdom study of the margin southwest of the English Channel last year.

\title{
Recent advances in polarized electron sources
}

IN his article "Polarized electrons are going around in circles" (News \& Views 283,$248 ; 1980$ ) C.B. Lucas notes that recent advances in sources of polarized electrons should provide insights into fields as diverse as neutral currents, surface physics, and molecular optical activity. He calls attention to recent developments in atomic physics-based polarized electron sources and to the fact that beams of electrons and positrons become polarized as they circulate in a storage ring. We would like to describe some very recent advances that have been made in the development and application of solid state sources of polarized electrons.

Extraction of electrons from a ferromagnetic solid offers a conceptually elegant source of polarized electrons. The electrons are aligned by an external magnetic field and exist in densities unobtainable in aligned atom beams. A very useful source has been demonstrated ${ }^{1}$ using field emission from a tungsten tip that has been coated with europium sulphide. At the operating temperature of $10 \mathrm{~K}$, the EuS is ferromagnetic and acts as a spin filter. A polarization of 0.85 is obtained at currents up to $10 \mathrm{nA}$. Most notably, the source has very high electron optical brightness and will be useful in applications requiring highly collimated, highly focused beams.

The most recently developed ${ }^{2}$ solid state source of polarized electrons uses photoemission from gallium arsenide. When GaAs is irradiated with circularly polarized light, the electrons excited from the maximum of the spin-orbit-split valence band may be polarized up to a theoretical value of $50 \%$. GaAs is one of those special materials in which, by application of caesium and oxygen to the surface, the vacuum level can be lowered below the bulk conduction band minimum to produce the so-called 'negative electron affinity' emitter. Negative electron affinity GaAs is not only an extremely efficient photoemitter but the electrons can be polarized! When the light is modulated from right to left circular polarization, the direction of the polarization of the electron beam is also modulated, facilitating the use of very sensitive signal detection techniques.

A source of polarized electrons utilizing GaAs has been built which produces a continuous beam with a polarization of $\pm 43 \%$, and a beam

\footnotetext{
1. Kisker, E., et al. Phys. Rev. B18, 2256 (1978) and

Pierce, D.T. \& Meier, F. Phys. Rev. B13, 5484 (1976); Pierce, D.T., Wang, G. C. \& Celotta, R.J. Appl. Phys. Lett. 3, 220 (1979); Pierce, D.T. et al. Rev. Sci. Instrum. 51. April 1980.

3. Prescott, C.Y. et al. Phys. Lett. 77B, 347 (1978)

4. Celotta, R.J. et al. Phys. Rev. Lett. 43, 728 (1979).

Wang, G.-C., et al. Phys. Rev. Lett. 42, 1349 (1979).
}

current of $20 \mu \mathrm{A}$ has been obtained for one milliwatt of incident circularly polarized light ${ }^{2}$. A pulsed GaAs source has produced microsecond pulses of several hundred milliamperes at a $120 \mathrm{~Hz}$ repetition rate ${ }^{3}$.

In a sense, the GaAs source is the solid state analogue of the source utilizing the 'Fano effect' in caesium discussed by Lucas. The higher density of the solid provides much more intense electron beams; typically three orders of magnitude more intense than existing Fano effect sources. The energy spread of the GaAs source is $130 \mathrm{meV}$ FWHM, and the effective source area is determined by the focus of the circularly polarized light, typically $0.5 \mathrm{~mm}$ diameter. The electron optical brightness is second only to the EuS/W field emission source.

The Fano effect in Cs can produce electron beams nearly $100 \%$ polarized. In most experiments the lower polarization of the GaAs source is easily compensated by increasing the intensity of the beam, but there are experiments, for example, where target damage is a problem, where higher polarization is required. There is hope of increasing the polarization of the GaAs source by lifting the degeneracy at the valence band maximum by (1) stressing the crystal (2) preparing epitaxially grown multilayer structures to confine the electron in one-dimensional potential wells, or by using (3) crystal field splitting in chalcopyrite semiconductors such as $\mathrm{CdSiAs}_{2}$ or $\mathrm{CdGeP}_{2}$, which are ternary analogues to GaAs.

The GaAs source has already been very effectively applied at SLAC to measure the parity non-conservation in inelastic scattering of $20 \mathrm{GeV}$ polarized electrons from hydrogen and deuterium ${ }^{3}$. It has been used to observe the surface magnetization of ferromagnetic nickel through the exchange interaction in low energy polarized electron scattering experiments ${ }^{4}$. It has also been used in polarized electron diffraction studies of single crystal tungsten 5 . The recent demonstrations of the characteristics of this type of source have led to the construction of such devices by nearly every group engaged in polarized electron scattering.

We agree completely with Lucas that there are now many exciting research opportunities because of recent advances in polarized electron sources. We believe that the new solid state source technology will play a significant part.

DANIEL T. PIERCE

ROBERT J. CELOTTA

US National Bureau of Standards,

Washington DC 20274 